\title{
A Digital Twin BaSed Self-Calibration Tool For Fault PREDiCTION OF FDM AdDitive ManUfaCtURING SySTEMS
}

\author{
Fabio Corradini \& Marco Silvestri
}
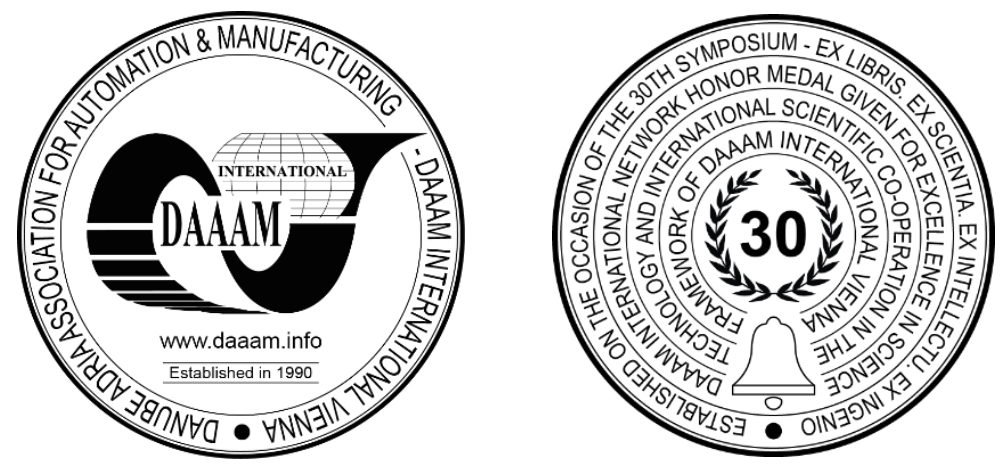

This Publication has to be referred as: Corradini, F[abio] \& Silvestri, M[arco] (2021). A Digital Twin Based SelfCalibration Tool for Fault Prediction of FDM Additive Manufacturing Systems, Proceedings of the 32nd DAAAM International Symposium, pp.0607-0616, B. Katalinic (Ed.), Published by DAAAM International, ISBN 978-3-90273433-4, ISSN 1726-9679, Vienna, Austria

DOI: $10.2507 / 32$ nd.daaam.proceedings.086

\begin{abstract}
Among the advantages of introducing digital twins on production systems, there is the ability to identify their eventual critical state and to enable predictive maintenance policies. The failure of a manufacturing process, in general, can be anticipated in phase of simulation, if tied up to wrong settings, or in phase of operation, if tied up to environmental variables. In both cases, knowing the conditions that could cause the failure allows to intervene in a timely and effective manner. Here a method is proposed to explore the system operating parameters in a systematic way: the system is able to process signals collected in real time by machine's sensors and to reproduce both the trajectories of the moving parts and the material deposition process. This also makes possible to predict manufacturing tolerances that will be obtained. On a FDM Cartesian 3D printer a self-calibration procedure is used to find the maximum torque that can be delivered by the drives at different speeds in an automatic and repeatable way to find the maximum speed and acceleration at which the machine can operate safely. Additional accelerometers were installed on the machine to validate the adopted procedure: tests results demonstrate the effectiveness of the system.
\end{abstract}

Keywords: self-calibration; digital twin; additive manufacturing; fault prediction

\section{Introduction}

In modern manufacturing plants, the use of digital twins offers significant advantages. A digital twin is a multiphysics digital simulation directly connected to its real counterpart, which can be a plant, a machine, or a product, through sensors, interfaces, and electronic control systems [1], [2]. The advantages offered using a digital twin can be of various nature [3]:

- Simplified monitoring of working parameters and automatic management of data flows.

- Real-time or offline optimization of work settings.

- Prediction and prevention of machining errors.

One of the fields where digital twins have proven to be particularly useful is in manufacturing machining, particularly additive manufacturing. In fact, since additive manufacturing is a relatively recent technology based on complex physical phenomena, it can benefit greatly from the ability to collect and organize machining data in a rational and automatic way. 
Moreover, it must be considered that additive manufacturing highest costs are mainly related to raw material and machining time, so the optimization of production process becomes even more important [4]. Over time, there have been several proposals for digital twins for additive manufacturing, most often referring to metal 3D printers [5], [6], [7], [8], [9], as the cost of raw material better justifies the expense of optimizing the process. Many works were also proposed on FDM 3D printers as they are very popular and easily available [10], [11], [12], [13], [14], [15], [16].

One of the problems encountered in the realization of digital twins is the correct characterization of the physical system, in fact the digital model on which the operation of the system is based must be calibrated to correctly perform the procedures of optimization and error prediction. The need to overcome this difficulty is at the basis of the rationale for the study presented here.

With reference to the prediction of errors, it was decided to carry out a series of tests that would force a prejudicial behaviour for the processing in progress, without damaging the machine, in order to understand the operating limits of the system and, using the physical model of the digital twin, characterize some relevant aspects of the model itself. In practice, by varying the input data to the real machine, the dual purpose of identifying the precise value of the harmful input and characterizing the physical model in a more appropriate way is achieved.

\section{Material and Methods}

The concept exposed above is applied to the error prediction function of a digital twin of an FDM 3D printer. In laboratory Cartesian FDM printers, two of the most common reasons for failure of a machining process are layer mismatch or extruder cogging. Both problems can be traced back to the stepper motors that misses steps while driving the corresponding axes and they can be greatly reduced by carefully choosing the printing settings (maximum speeds and accelerations). The problem is that choosing conservative kinematic limits can also greatly increase the machining time, with consequent increase in costs.

The correct calibration of the kinematic parameters can be carried out by the digital twin, thanks to its physical model of the machine, but it is necessary to know both the pull-out curve of the stepper motors and the dynamic behaviour of the system, including non-linear effects. The torque curve of stepper motors is usually provided by the manufacturer, but the test conditions are almost never equivalent to the working conditions (different drivers, micro-steps settings, power supply). It is possible to obtain the curve using a test bench (that is not always available), or through analytical calculations [17] or software simulations [18], [19], solutions that require in any case the knowledge of the internal characteristics of the motor.

To overcome this problem, an algorithm has been devised that generates a series of trajectories capable of stressing the motors with increasing speed and loads. Using the sensors installed on the machine, it is possible to monitor the response of the system and in this way not only detecting critical accelerations and speeds under load conditions, but also deducing the pull-out curve of the motors.

\section{1. $3 D$ printer and sensors}

The FDM 3D printer used as a test is a Cartesian type, built specifically for printing large parts (Fig. 1). The printer features an extruder block that is movable in both $\mathrm{X}$ and $\mathrm{Y}$ directions and houses two extruders with motors. The motion of the extruder block is provided by two NEMA 17 42BYGHW811 stepper motors for the X-axis and a motor of the same type for the $\mathrm{Y}$-axis. Both actuators operate via toothed belt drive with a $40 \mathrm{~mm}$ circumference pulley. The $\mathrm{X}$-axis motors are connected in parallel to the same MKS TB6600 driver powered at $12 \mathrm{~V}$ and set to a maximum current of $3 \mathrm{~A}$, the $\mathrm{Y}$ axis motor is connected to a driver of the same type but set to $1.5 \mathrm{~A}$. The printing table is mobile along the $\mathrm{Z}$ axis in vertical direction, has a usable area of 626 X $355 \mathrm{~mm}$, and can be heated to improve adhesion.

The extruders are direct drive type, suitable for receiving a $1.75 \mathrm{~mm}$ filament, whose feeding is obtained through a pinion with $40 \mathrm{~mm}$ circumference. Brass nozzles have an inner diameter of $0.4 \mathrm{~mm}$. The extruders motor is a NEMA 17 42BYGHW811B dual shaft and is powered by an A4988 driver also at 12V. All motors are controlled with 1/16 pitch micro-steps. Details about operation and sensors of Z-axis are not provided because in this type of machine its calibration has almost no impact on the printing speed. The printer controller is an Arduino Mega board with RAMPS 1.4 expansion board, on the microcontroller is installed the firmware Marlin v 1.1.9 [20].

To provide the digital twin with feed-back data, the system has been equipped with various types of sensors, the most important of these are optical encoders that measure axis position. X and Y axes are monitored by two ENS1J-B28 L00256L encoders placed on the idler pulley, the system has a resolution of 20.477 pulse/mm referred to the extruder feed. For testing purposes, only one of the extruders was kept active, the feedback is given by an encoder of the same type mounted on the rear shaft of the E-axis motor, the resolution obtained is 25.6 pulses/mm referred to the filament feed. Other sensors have also been installed, although they are not relevant in the axis calibration procedure, among them an encoder for the Z-axis, two additional thermistors and a computer-vision tracking system using a video camera and a dedicated processor.

The printer is also equipped with 6 EPOSMote III smart sensors [21] to collect data from the other sensors and to send them to the interface of the digital twin. In addition, they provide an LSM330 integrated accelerometer. The data coming from the accelerometer of the sensor placed on the extruder block will be used as reference for the tests performed on $\mathrm{X}$ and $\mathrm{Y}$ axes. 
The digital twin core operates on a workstation placed next to the printer and receives the data from the smart sensors via USB serial connection.

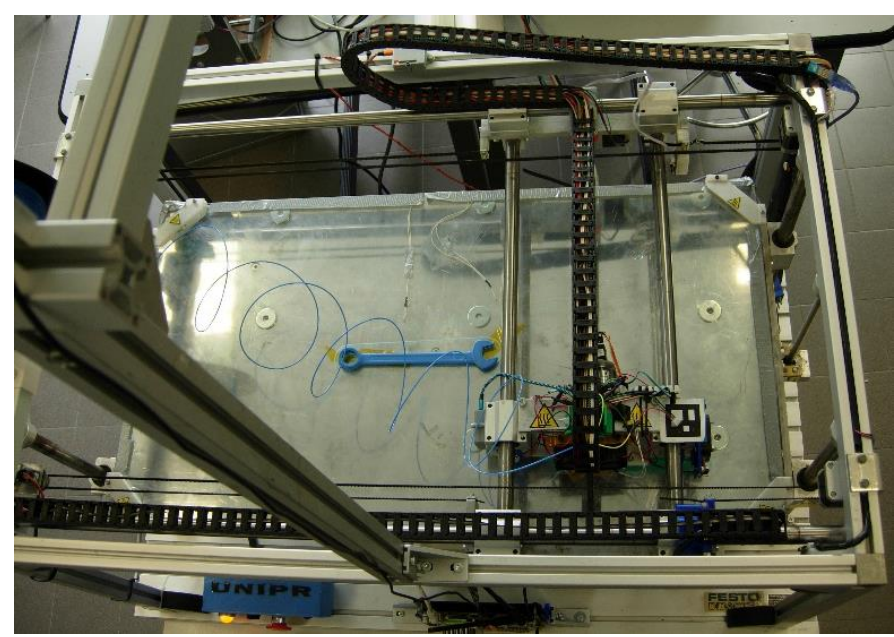

Fig. 1. Printer used for test

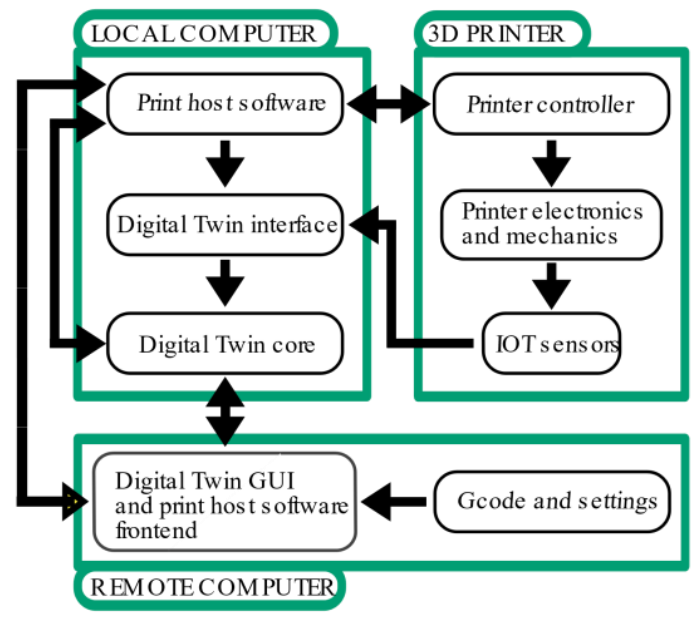

Fig. 2. Digital twin scheme

\subsection{The digital twin}

The digital twin is a complex system that contains various intercommunicating parts (Fig. 2). The main parts are the print host software, the interface, the core, and the GUI:

- The digital twin interface is a python script that operates locally on a computer near the printer. It is responsible for receiving data from the smart sensors and the digital vision system and sending it to the digital twin core. It is also able to receive from the print host software the information related to the printing in progress. It additionally performs the task of synchronizing the timestamps at system start-up and sending the collected data to a cloud repository from where it can be accessed or used for other projects.

- The print host software is not technically part of the digital twin but is used by it to act on processes in progress. This program performs the task of receiving commands from user (even remotely) and forward them to the printer controller via serial connection. In this system, this program no longer interacts directly with the user but receives commands and sends information both to the core and to the GUI of the digital twin. A version of Octoprint [22] was used as print host software since it offers a practical plugin system to make changes and an API to manage the printer remotely without a graphical interface.

- The digital twin core is an application programmed in python and $\mathrm{C}++$ that operates on a workstation located near the printer running the model of the physical system. It performs various functions including, error detection, calculation of significant parameters for printing, offline simulation of printing processes, calculation of wear and tear of various components, real-time building of the virtual model of the printed part.

- The GUI is the part of the program dedicated to interaction with the end user, it can also run on a remote device and be used for remote control. The GUI has the basic function of allowing user to launch prints, keep the current process monitored and view the results of old jobs. It can also perform more advanced task such as allowing to initiate wizards to recover from an error situation and performing post processing of the data collected during printing to identify deviations between the model of the printed part and its CAD representation.

\subsection{The calibration procedure}

One of the main reasons that can lead to 3D printing failure is when one of the stepper motors misses one or more steps. This event occurs when the torque exerted by the motor at the current speed is insufficient to support the load, in such case the motor shaft cannot move forward by the commanded pitch and consequently cannot maintain the motion profile set.

To determine the conditions that cause this damaging event, a series of trajectories have been designed to gradually increase the speed and load required for the axes whose operation is more critical. During the execution of the movements, the digital twin of the machine is kept active so that, by acquiring displacements and accelerations, it is able to determine the critical values and, through its physical model, to give an estimate of the maximum loads the motors can bear and of their pull-out curve.

This operation has not been conceived to be performed normally during the operation of the printer and the digital twin but only during its set-up or after a relevant hardware modification (change of moving masses, replacement of the motor, driver, or nozzle). For this reason, the calibration procedure cannot be managed remotely from the GUI but must be launched directly at the start-up of the digital twin core of which it is an integral part. 
The calibration procedure operates on the most significant axes for printing purposes, namely the X, Y, and E axes. The $\mathrm{Z}$ axis has been excluded because on machines of this type it plays a very marginal role, limiting itself to moving a few tenths of a millimetres at the end of each layer. The calibration procedure uses the connection of the digital twin core with the print host program to send to the printer controller the g-code and m-code lines containing the information necessary to execute the desired profile (point of arrival, maximum speed, and acceleration), then the feedback of the encoders is used to evaluate the outcome of the test. The printer controller was set to perform trapezoidal speed profiles with constant acceleration transients.

\subsubsection{The procedure for $X$ and $Y$ axes}

Based on the structure of the machine, it can be said that the main load acting on the $\mathrm{X}$ and $\mathrm{Y}$ axes is dynamic, due to the high mass of the extruder block. For this reason, the trajectories that these axes must follow will be characterized by increasing acceleration values, so as to gradually increase the load. At the same time the maximum speed is also increased since it is known that at high speeds the torque that can be delivered by the motors decreases. The trajectories for these axes have been generated to have trapezoidal speed profiles and satisfying the following criteria:

- The maximum usable space depends on the length of the axis, but a safety margin must always be maintained between the end of the movement and the limit switch so that a failure in the braking phase does not cause damage.

- The acceleration and deceleration phase cannot be longer than $8 \mathrm{~s}$, as this causes problems to the printer controller firmware (overflow of a timer causes irregular movements).

- The acceleration must be maintained for a sufficient distance and overcome the backlash and elasticity of the kinematic chain; in this way it can be sure that the motor is subject to the desired load for a certain number of steps.

- The maximum speed allowed for a test at a given acceleration must be in any case lower than the one that caused a failure at a lower acceleration.

- After the acceleration ramp the axis maintains the set speed for a certain time. A maximum value relative to this time is set to reduce the duration of the test.

To satisfy these conditions for each acceleration $a_{i}$ set, a series of maximum speeds must be tested between $v_{\text {inf }}$ and $v_{\text {sup }}$ calculated in this way:

$$
\begin{aligned}
& v_{\text {inf }}=\sqrt{2 \cdot s_{\text {acc min }} \cdot a_{i}} \\
& v_{\text {sup }}=\min \left(v_{\max }, a_{i} \cdot t_{\max }, a_{i} \sqrt{\frac{s}{a_{i}}}\right)
\end{aligned}
$$

Where $v_{\max }$ is the lowest speed at which a failure was detected, $a_{i}$ is the acceleration of the profile, $t_{\max }$ is the maximum possible duration for the acceleration and deceleration phase, $s$ is the space available to perform the test, $s_{\text {acc min }}$ is the minimum space for which the acceleration phase must extend. For each acceleration $a_{i}$ it is then possible to execute $\mathrm{n}$ tests at different speeds dividing the interval between $v_{\text {inf }}$ and $v_{\text {sup }}$ in steps of about $80 \mathrm{~mm} / \mathrm{s}$. Of course, if $v_{\text {sup }}<v_{\text {inf }}$ it is not possible to execute any test for the corresponding acceleration and a new acceleration is calculated using (3).

$$
a_{\text {new }}=\frac{v_{\text {sup }}{ }^{2}}{s_{\text {acc } \min }}
$$

Once the trajectory for each test is defined, it is possible to calculate the time needed to execute it. Given a certain trajectory the test is considered passed if the assigned law of motion is executed in the required time both in the outward and in the return phase (i.e., the same trajectory is executed twice, forward and backward, so that at the end of the test the axes are in the initial configuration). In practice the law of motion is considered executed if the feedback position signal is close enough to the target position for enough time. More precisely, if the difference between the actual position measured by the encoders and the target position remains less than a threshold value (settle window) for at least a certain time (settle time). If this condition does not occur within a time equal to 1.5 times the time theoretically required to make the movement, it is considered that the axis was not be able to reach the target or has exceeded it by failing to decelerate.

If a test is passed at a speed $v_{j}$ it is repeated at speed $v_{j+1}$ until the $v_{s u p}$ for the current acceleration is reached. At this point a new set of trial is started at a higher acceleration (twice the previous one). If the test is not passed, two conditions can occur, if $\mathrm{j}=0$ (i.e., $v_{j}=v_{\text {inf }}$ ) the motor has failed the movement at the minimum possible speed, therefore the current acceleration is considered the maximum bearable by the motor (corresponding to the holding torque) and the test for the current axis is concluded. If, instead, the test fails for $v_{j+1}>v_{i n f}$, then $v_{j}$ is set as the limit speed for the current acceleration, the variable $v_{\max }$ is updated and a new phase with higher acceleration is performed. In this way in any case the tests performed at higher acceleration cannot exceed the speed that caused the failure of tests with lower accelerations. This algorithm is shown in Fig 3. After a series of tests the parameters of the algorithm were fixed according to Table 1. 


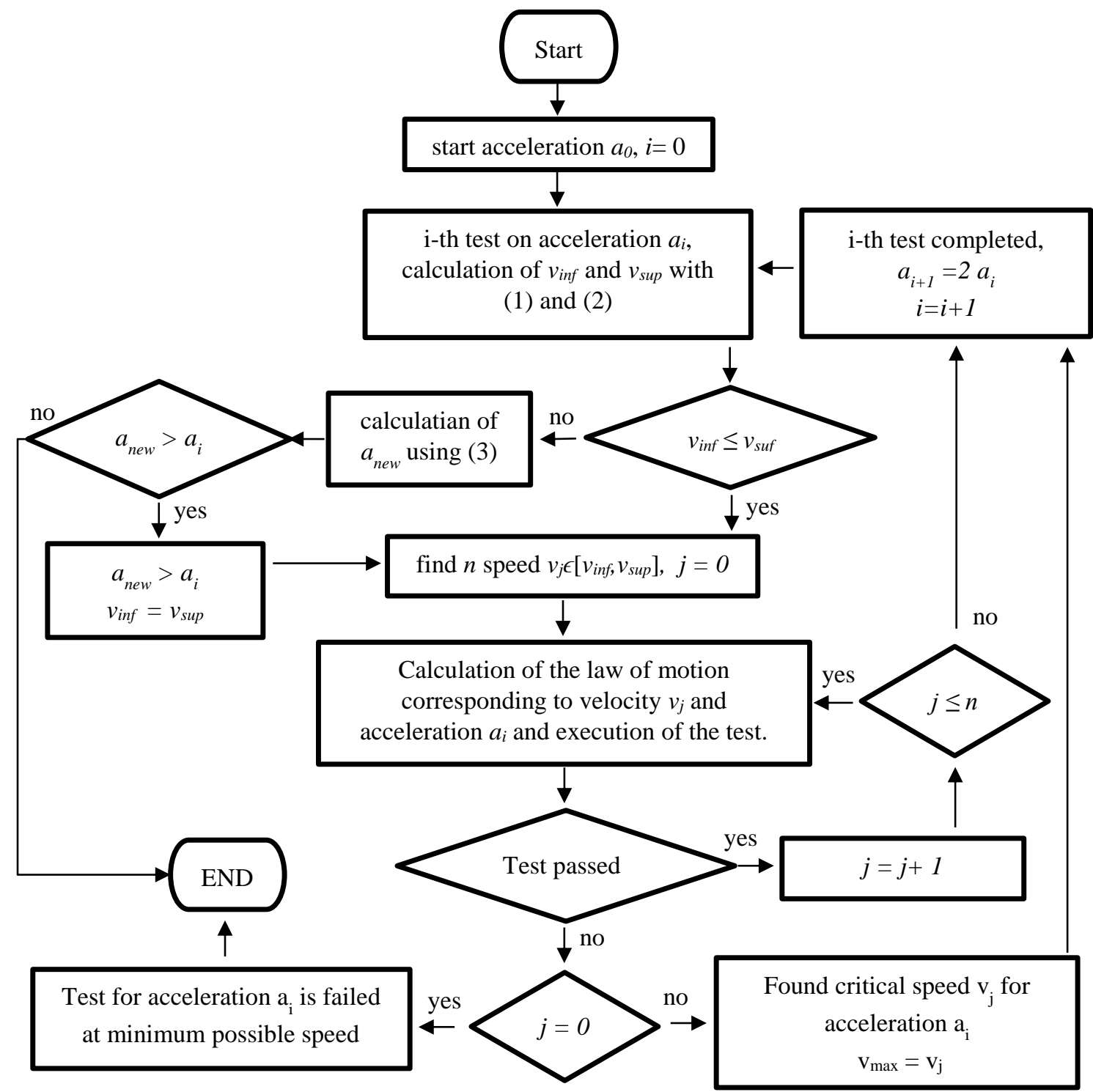

Fig. 3. Flowchart diagram explaining algorithm for calibration of $\mathrm{X}$ and $\mathrm{Y}$ axes. Every failed test is recorded.

\subsubsection{The procedure for E-axis}

Regarding the E-axis, the main load to which the motor is subjected is viscous and therefore dependent on extrusion speed rather than acceleration. This test is very useful since the maximum printing speed greatly affects the processing speed. To perform this test, the extruder is first brought to the most suitable working temperature for PLA to achieve dimensional accuracy $\left(200^{\circ} \mathrm{C}\right)$ [23], then a given amount of material is extruded at constant speed. If the extrusion succeed is retried with a greater speed (every round the speed is increased by a $\mathrm{k}$ factor).

When, by means of the encoder mounted on the extruder shaft, an occlusion is detected, the test is repeated with halved acceleration but maintaining the speed that caused the failure so as to exclude any influence of the acceleration. This is repeated until the acceleration fall below threshold value $a_{\min }$. The algorithm, shown in Fig 4, is simpler than that for the $\mathrm{X}$ and $\mathrm{Y}$ axes. Table 2 shows the data used in the testing phase. For the test to be valid it is necessary to set an amount of extruded filament $s$ such that the compressibility of the material is overcome. To ensure the reliability and repeatability of the results is necessary to tighten the screw that adjusts the force with which the pinion presses on the filament to prevent its slippage. 


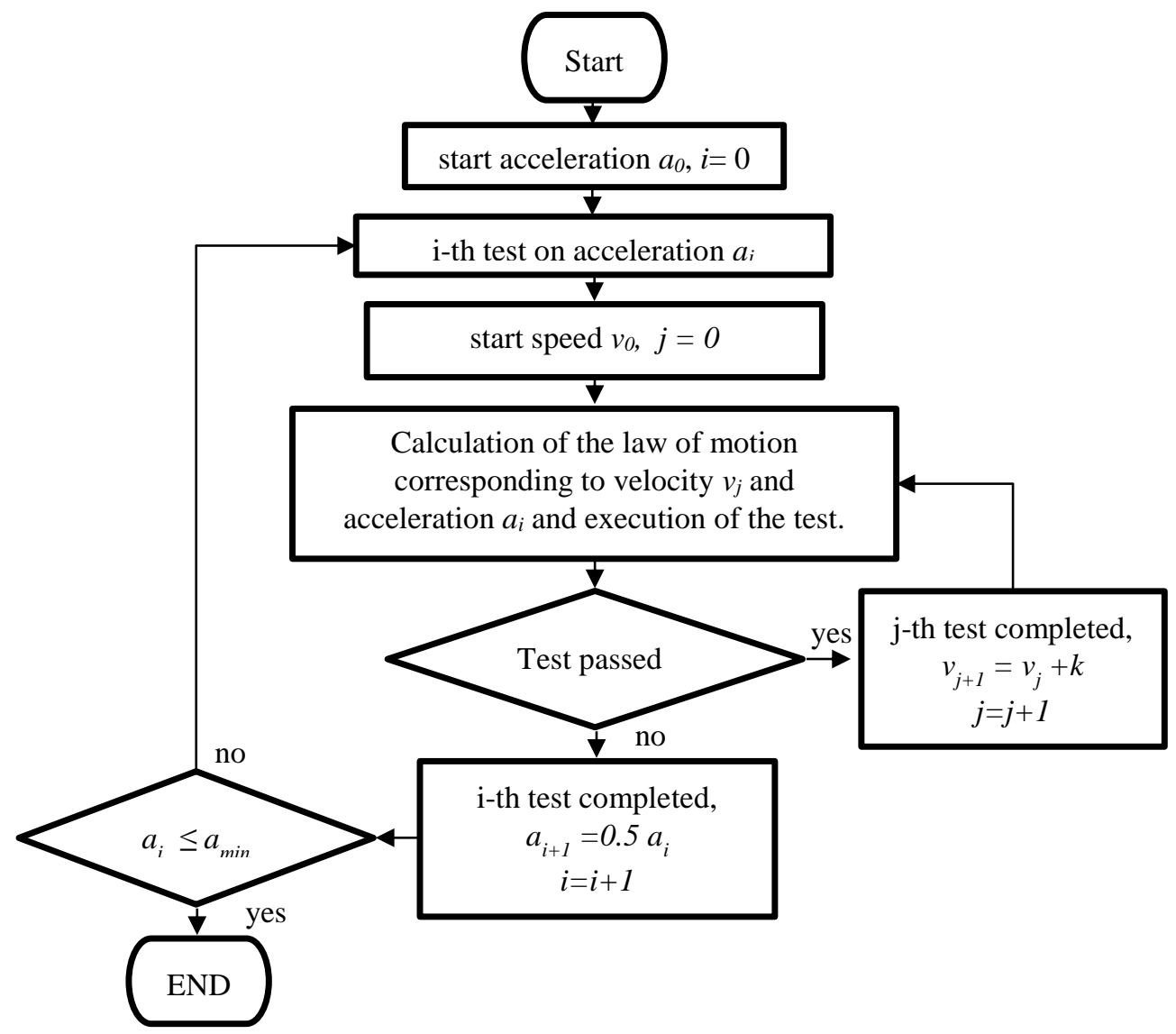

Fig. 4. Flowchart diagram explaining algorithm for calibration E-axis. Every failed test is recorded.

\begin{tabular}{|c|c|c|}
\hline Parameter & X-axis & Y-axis \\
\hline $\mathrm{s}$ & $500 \mathrm{~mm}$ & $300 \mathrm{~mm}$ \\
\hline$t_{\max }$ & $8 \mathrm{~s}$ & $8 \mathrm{~s}$ \\
\hline$s_{\text {acc } \min }$ & $5 \mathrm{~mm}$ & $5 \mathrm{~mm}$ \\
\hline$a_{0}$ & $16 \mathrm{~mm} / \mathrm{s}^{2}$ & $16 \mathrm{~mm} / \mathrm{s}^{2}$ \\
\hline$t_{\text {max const speed }}$ & $1 \mathrm{~s}$ & $1 \mathrm{~s}$ \\
\hline Settle window & $1 \mathrm{~mm}$ & $1 \mathrm{~mm}$ \\
\hline Settle time & $0.1 \mathrm{~s}$ & $0.1 \mathrm{~s}$ \\
\hline
\end{tabular}

\begin{tabular}{|c|c|}
\hline Parameter & E-axis \\
\hline$a_{0}$ & $10000 \mathrm{~mm} / \mathrm{s}^{2}$ \\
\hline$v_{0}$ & $2.090 \mathrm{~mm} / \mathrm{s}$ \\
\hline$s$ & $40 \mathrm{~mm}$ \\
\hline$a_{\min }$ & $1250 \mathrm{~mm} / \mathrm{s}^{2}$ \\
\hline$k$ & $1.045 \mathrm{~mm} / \mathrm{s}$ \\
\hline Settle window & $0.5 \mathrm{~mm}$ \\
\hline Settle time & $0.1 \mathrm{~s}$ \\
\hline
\end{tabular}

Table 1. Parameters for tests on $\mathrm{X}$ and $\mathrm{Y}$ axes

Table 2. Parameters for tests on E axes

\section{Results and discussion}

Tests performed and the results obtained for the $\mathrm{x}$ and $\mathrm{y}$ axes are reported:

\begin{tabular}{|c|c|c|c|c|c|c|c|}
\hline Acceleration [mm/s2] & \multicolumn{7}{|c|}{ max speed [mm/s] } \\
\hline 16 & 12.649 & 89.443 & & & & & \\
\hline 32 & 17.889 & 72.190 & 126.491 & & & & \\
\hline 64 & 25.298 & 102.092 & 178.885 & & & & \\
\hline 128 & 35.777 & 108.179 & 180.581 & 252.982 & & & \\
\hline 256 & 50.596 & 127.390 & 204.184 & 280.977 & 357.771 & & \\
\hline 512 & 71.554 & 143.956 & 216.358 & 288.759 & 361.161 & 433.563 & $\mathbf{5 0 5 . 9 6 4}$ \\
\hline 1024 & 101.193 & 168.655 & 236.117 & 303.579 & 371.041 & $\mathbf{4 3 8 . 5 0 3}$ & \\
\hline 2048 & 143.108 & 216.957 & 290.805 & 364.654 & 438.503 & & \\
\hline 4096 & $\mathbf{2 0 2 . 3 8 6}$ & & & & & & \\
\hline
\end{tabular}

Table 3. Test performed on $\mathrm{X}$-axis, in bold are reported the failed tests. 


\begin{tabular}{|c|c|c|c|c|c|c|c|}
\hline Acceleration [mm/s2] & \multicolumn{7}{|c|}{ max speed $[\mathrm{mm} / \mathrm{s}]$} \\
\hline 16 & 12.649 & 69.282 & & & & & \\
\hline 32 & 17.889 & 57.934 & 97.980 & & & & \\
\hline 64 & 25.298 & 81.931 & 138.564 & & & & \\
\hline 128 & 35.777 & 89.171 & 142.565 & 195.959 & & & \\
\hline 256 & 50.596 & 126.107 & 201.618 & 277.128 & & & \\
\hline 512 & 71.554 & 135.627 & 199.700 & 263.773 & 327.846 & 391.918 & \\
\hline 1024 & 101.193 & 176.703 & 252.214 & 327.725 & 403.235 & 478.746 & \\
\hline 2048 & 143.108 & 210.236 & 277.363 & 344.491 & 411.618 & 478.746 & \\
\hline 4096 & 202.386 & 271.476 & 340.566 & 409.656 & 478.746 & & \\
\hline 8192 & 286.217 & 350.393 & 414.569 & & & & \\
\hline 16384 & 404.772 & & & & & & \\
\hline
\end{tabular}

Table 4. Test performed on Y-axis, in bold are reported the failed tests.

For reasons of space, it is not possible to report the data collected during each test, therefore it is reported only an example of successful test and one of failed test for the $\mathrm{X}$ axis. Figure 5 (a) shows the data taken by the encoder during the test at acceleration of $512 \mathrm{~mm} / \mathrm{s}^{2}$ and maximum speed of $361.161 \mathrm{~mm} / \mathrm{s}$. The speed data were obtained by deriving the position values and using a moving window filter. The acceleration data were obtained by deriving and filtering the speed data (hence also they are derived from the encoders), but, for validation purposes, accelerometers were mounted on machine and their measured values show a good agreement with the calculated ones. So, it is demonstrated that future applications could rely on the use of encoders alone. From the graphs it can be seen that the assigned joint trajectory is respected but executed with a certain delay (about $0.2 \mathrm{~s}$ ), due to the fact that when the motion command is sent (and the timer starts to run) the digital twin core must send the instructions to the host software via TCP/IP connection. This in turn forwards the message via serial connection to the printer controller that reads and processes it. Further delays are given by the time needed to activate the drivers and magnetize the motor windings and by the effect of mechanical backlashes and belt elasticity.

Figure (b) shows the same data but related to a test in which the motor misses some steps (acceleration of $512 \mathrm{~mm} / \mathrm{s} 2$ and speed of $505.964 \mathrm{~mm} / \mathrm{s}$ ). In the graph it can be clearly seen that at the instant $1.08 \mathrm{~s}$, almost at the end of the acceleration phase, the movement suddenly stops in an uncontrolled way and then resumes later at a lower speed in the return phase. The abrupt stop and subsequent sudden restart create a considerable impulsive force that affects the structure of the printer making it oscillates on its support (this oscillation is clearly visible in the accelerometer trace).

Result of E-axis test are also reported:

\begin{tabular}{|c|c|c|c|}
\hline Acceleration $[\mathrm{mm} / \mathbf{s} 2]$ & \multicolumn{3}{|c|}{ max speed $[\mathrm{mm} / \mathbf{s}]$} \\
\hline 10000 & 2.090 & 3.135 & $\mathbf{4 . 1 8 0}$ \\
\hline 5000 & $\mathbf{4 . 1 8 0}$ & & \\
\hline 2500 & $\mathbf{4 . 1 8 0}$ & & \\
\hline
\end{tabular}

Table 5. Test performed on E-axis, in bold are reported the failed tests.
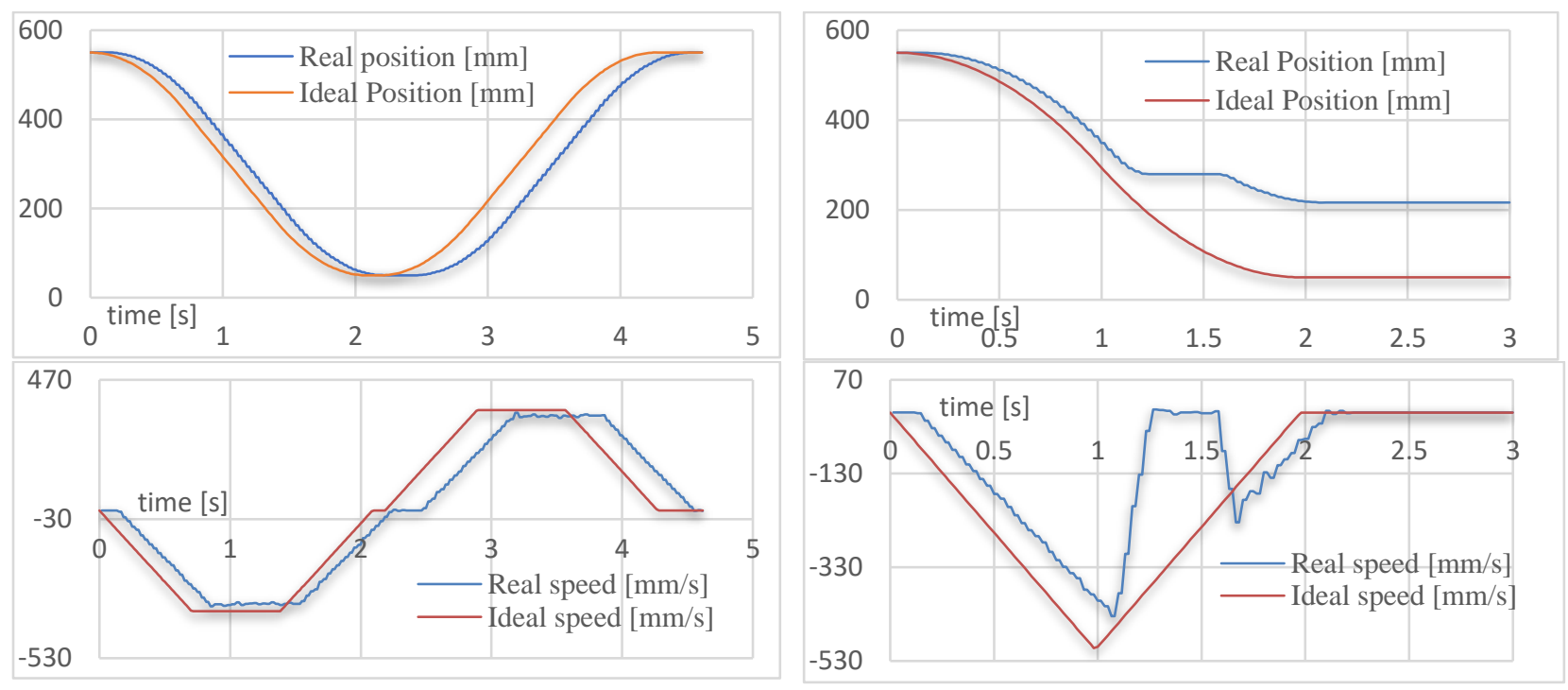


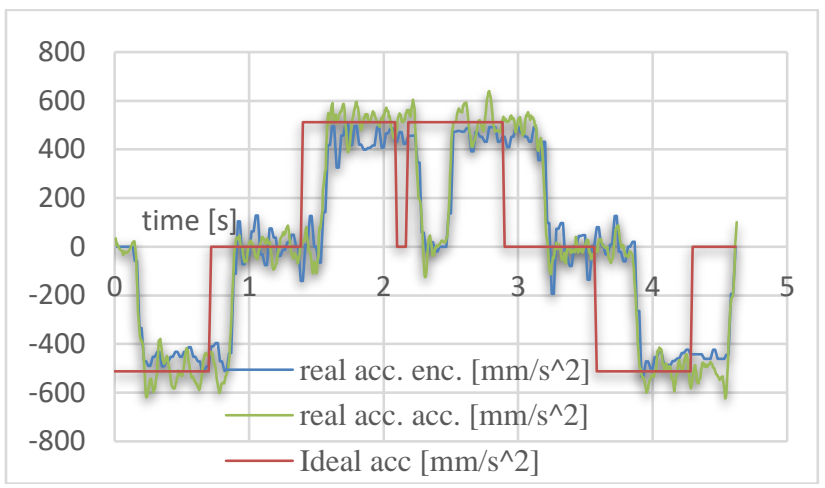

(a)

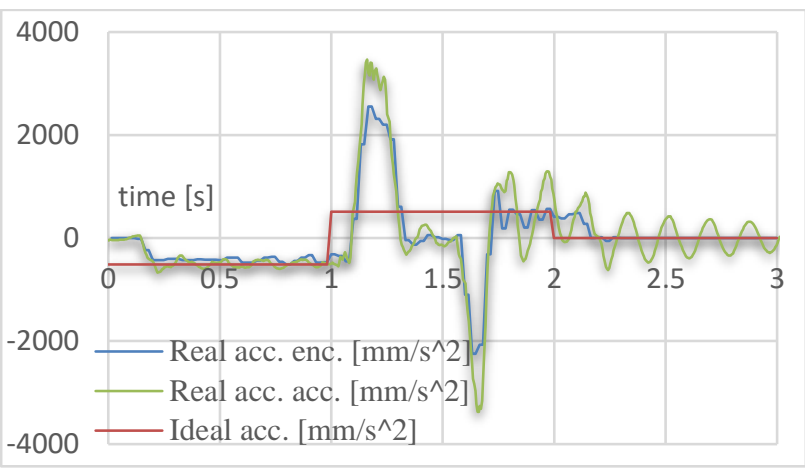

(b)

Fig. 5. Figure (a) represents position, speed, and acceleration of a successful test $\left(\mathrm{X}\right.$-axis, acc. $=512 \mathrm{~mm} / \mathrm{s}^{2}, \mathrm{max} \mathrm{speed}$ $=361.161 \mathrm{~mm} / \mathrm{s})$; Figure (b) represents the same measure for a failed test $\left(X\right.$-axis, acc. $=512 \mathrm{~mm} / \mathrm{s}^{2}$, max speed $=$ $505.964 \mathrm{~mm} / \mathrm{s}$ )

In Fig 6 can be seen the E-axis motor missing steps at least 4 times, at each time is visible a backflow of material pushing back the pinion, a phenomenon due to the elasticity of the molten polymer and the nozzle pipe.

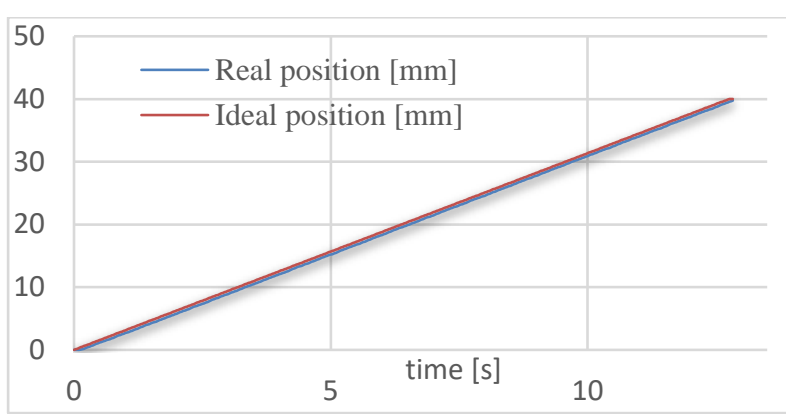

(a)

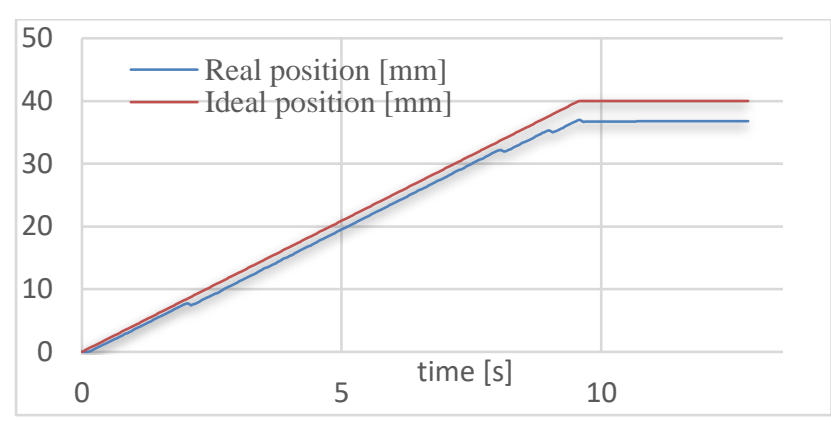

(b)

Fig. 6. Figure (a) represents the position of a successful test $\left(\right.$ E-axis, acc. $\left.=10000 \mathrm{~mm} / \mathrm{s}^{2}, \max \mathrm{speed}=3.135 \mathrm{~mm} / \mathrm{s}\right)$;

Figure (b) represents the same measure for a failed test (E-axis, acc. $=10000 \mathrm{~mm} / \mathrm{s}^{2}$, $\max$ speed $=4.180 \mathrm{~mm} / \mathrm{s}$ )

Once the tests are finished it is possible to order the values where failure occurred at increasing speed, for this purpose the point with the highest acceleration is considered at zero speed as it is the point that best approximates the static torque of the motor. Considering that the mass of the X-axis moving carriage is $10.82 \mathrm{~kg}$ while that of the $\mathrm{Y}$-axis is $2.43 \mathrm{~kg}$, neglecting all non-inertial loads the pull-out curves for the analysed motors can be obtained. They are shown in Fig. 7 and compared with the factory curve of a motor of similar size. It can be seen that the curve obtained is always lower, although of the same order of magnitude, this may be due to differences in the power circuit and to the fact that the implemented physical model does not take into account the friction forces and therefore underestimates the actual load acting on the motor. It can also be seen that the motors that move the X-axis have much lower performance than those of the Y-axis, this may be due to the fact that X-axis is driven by two motors operating in parallel, each with its own belt, and a certain load unbalancing could occur over time.

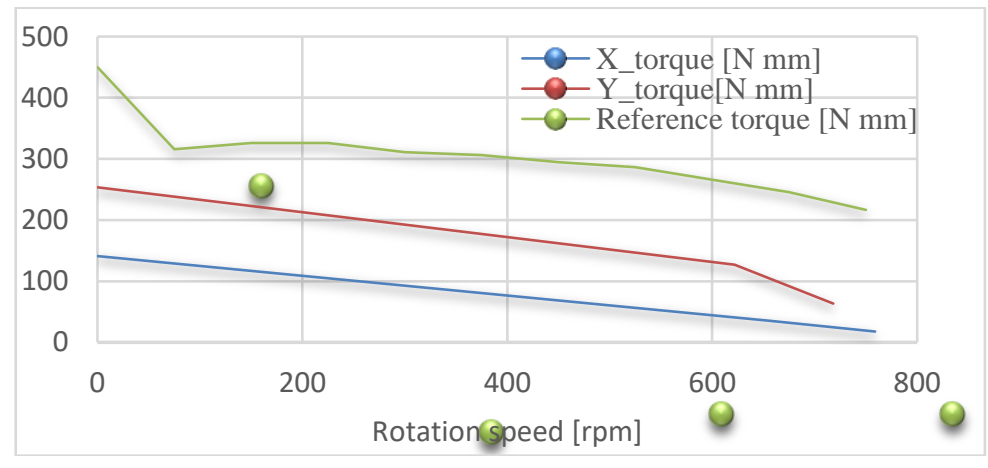

Fig. 7. X-axis and Y-axis motor pull-out curves compared to that provided by the manufacturer for a similar motor.

\begin{tabular}{|c|c|c|}
\hline Axis & $\begin{array}{c}\text { acc. } \\
{\left[\mathbf{m m} / \mathbf{s}^{2}\right]}\end{array}$ & $\begin{array}{c}\text { speed limit } \\
{[\mathbf{m m} / \mathbf{s}]}\end{array}$ \\
\hline $\mathrm{X}$ & 2048 & 292.33 \\
\hline$Y$ & 8192 & 414.57 \\
\hline $\mathrm{E}$ & 5000 & 4.180 \\
\hline
\end{tabular}

Table 6. Proposed settings for fast and safe machine movement 
As far as the load on the E axis is concerned, the speeds reached are not sufficient to draw a pull-out curve and therefore only the holding torque can be computed. The load is calculated from the physical model of the digital twin which models the nozzle as a capillary ending in an orifice. The pressure loss due to friction is calculated with the generalized Reynolds number for non-Newtonian fluids [24] in laminar regime using the properties of molten PLA [25], resulting in a holding torque of $268.94 \mathrm{~N} \mathrm{~mm}$ which is in line with what has been observed so far. Once the characterization of the motors has been obtained, it can be used to generate optimized working settings. The maximum acceleration that can be set for each axis will be half of the one corresponding to the maximum torque (to keep a safety margin) while the maximum speed will be that one corresponding to the torque found. The results for the series of tests just completed are shown in table 6.

\section{Conclusion}

An algorithm to calibrate the error prediction function of a 3D printer has been developed, implemented through a digital twin and tested in lab. Its use allows to optimize the settings of the machine and, at the same time, to characterize its operational behaviour. The procedure was applied to the prediction of layer mismatch and under-extrusion errors of a Cartesian-type FDM 3D printer. The calibration routine was defined to be carried out in a fully automatic way and provided as results the printing limit settings and the characterization of the motors under investigation. A solution of this type has the potential to be extended to different types of machinery and errors as long as they are repeatable and do not endanger the integrity of the machine or the safety of the operator. In the future is proposed to repeat the test using a model that takes into account friction and elastic parts of the machine in order to verify how a more accurate model can improve the results.

\section{References}

[1] Tao, F.; Zhang, H.; Liu, A. \& Nee, A. Y. C. (2019). Digital Twin in Industry: State-of-the-Art, IEEE Transactions on Industrial Informatics, Vol 15, No. 4, pp. 2405-2415., ISSN: 1551-3203, DOI: 10.1109/TII.2018.2873186.

[2] Sbaglia, L.; Giberti, H. \& Silvestri, M.(2019) 'The Cyber-Physical Systems within the industry 4.0 framework', Advances in Italian Mechanism Science - Mechanisms and Machine ScienceVolume 68, 2019, pp. 415-423, Springer Netherlands, DOI: 10.1007/978-3-030-03320-0_45

[3] Kostenko, D.; Kudryashov, N.; Maystrishin, M; Onufriev, V.; Potekhin, V. \& Vasiliev, A. (2018). Digital Twin Applications: Diagnostics, Optimisation and Prediction, Proceedings of the 29th DAAAM International Symposium, pp.0574-0581, B. Katalinic (Ed.), Published by DAAAM International, ISBN 978-3-902734-20-4, ISSN 1726-9679, Vienna, Austria DOI: 10.2507/29th.daaam.proceedings.083

[4] Frazier, W. E. (2014). Metal Additive Manufacturing: A Review., Journal of Materials Engineering and Performance, Vol. 23, No. 6, pp. 1917-1928. doi: 10.1007/s11665-014-0958-z.

[5] Knapp, G. L.; Mukherjee, T.; Zuback, J. S.; Wei, H. L.; Palmer, T. A.; De, A. \& DebRoy, T. (2017). Building blocks for a digital twin of additive manufacturing. Acta Materialia, Vol. 135, pp. 390-399. https://doi.org/10.1016/j.actamat.2017.06.039

[6] Sousa Agostino, Í. R., Morosini Frazzon, E.; Medeiros Fröhlich, A. A.; Silvestri, M.; Bornia, A. C.; Spengler, A.; Martina, J.; Fettermann, D. \& Tortorella, G. (2020). Perspectives for IoT-based integration of distributed and automated manufacturing lines for mass customization, Proceedings on 25th International Joint Conference on Industrial Engineering and Operations Management - IJCIEOM, Springer International Publishing, 2020, pp. 31 41, DOI: 10.1007/978-3-030-43616-2_4

[7] DebRoy, T.; Zhang, W.; Turner, J. \& Babu, S. S. (2017). Building digital twins of 3D printing machines. Scripta Materialia, Vol. 135, 119-124. https://doi.org/10.1016/j.scriptamat.2016.12.005

[8] Mandolla, C.; Petruzzelli, A. M.; Percoco, G. \& Urbinati, A. (2019). Building a digital twin for additive manufacturing through the exploitation of blockchain: A case analysis of the aircraft industry. Computers in Industry, Vol. 109, pp. 134-152. https://doi.org/10.1016/j.compind.2019.04.011

[9] Liu, C.; Le Roux, L.; Körner, C.; Tabaste, O.; Lacan, F. \& Bigot, S. (2020). Digital Twin-enabled Collaborative Data Management for Metal Additive Manufacturing Systems. Journal of Manufacturing Systems. https://doi.org/10.1016/j.jmsy.2020.05.010

[10] Balta, E. C.; Tilbury, D. M. \& Barton, K. (2019). A Digital Twin Framework for Performance Monitoring and Anomaly Detection in Fused Deposition Modeling. 2019 IEEE 15th International Conference on Automation Science and Engineering (CASE), pp. 823-829. https://doi.org/10.1109/COASE.2019.8843166

[11] Hu, L.; Nguyen, N. T.; Tao, W.; Leu, M. C.; Liu, X. F.; Shahriar, M. R. \& Al Sunny, S. M. N. (2018). Modeling of Cloud-Based Digital Twins for Smart Manufacturing with MT Connect. Procedia Manufacturing, Vol. 26, pp. 11931203. https://doi.org/10.1016/j.promfg.2018.07.155

[12] Chhetri, S. R.; Faezi, S.; Canedo, A. \& Faruque, M. A. Al. (2019). QUILT: Quality inference from living digital twins in IoT-enabled manufacturing systems. IoTDI 2019 - Proceedings of the 2019 Internet of Things Design and Implementation, pp. 237-248. https://doi.org/10.1145/3302505.3310085 
[13] Avventuroso, G.; Silvestri, M. \& Morosini Frazzon, E. (2018). Additive Manufacturing Plant for Large Scale Production of Medical Devices: A Simulation Study, IFAC-PapersOnLine, Vol. 51, No. 11, June 2018, pp. 14421447. ISSN: 2405-8963 https://doi.org/10.1016/j.ifacol.2018.08.312

[14] Cai, Y.; Wang, Y. \& Burnett, M. (2020). Using augmented reality to build digital twin for reconfigurable additive manufacturing system', Journal of Manufacturing Systems, Vol. 56, pp. 598-604. doi: 10.1016/j.jmsy.2020.04.005.

[15] Moretti, M.; Rossi, A. \& Senin, N. (2021). In-process monitoring of part geometry in fused filament fabrication using computer vision and digital twins, Additive Manufacturing, Vol. 37, p. 101609. doi: 10.1016/j.addma.2020.101609.

[16] Avventuroso, G.; Foresti, R.; Silvestri, M. \& Morosini Frazzon, E. (2017). Production paradigms for additive manufacturing systems: A simulation-based analysis, 2017 International Conference on Engineering, Technology and Innovation (ICE/ITMC), Madeira Island, Portugal, 2017, pp. 973-981. doi: 10.1109/ICE.2017.8279987

[17] Russell, A. P. \& Pickup, I. E. D. (1982). Calculation of the pull-out torque of hybrid stepping motors in the half-step mode, Electric Machines and Power Systems, Vol. 7, No. 2, pp. 103-114. doi: 10.1080/03616968208955440.

[18] Mariolo, L.; Rubino, A.; Spelta, D. \& Frosini, L. (2021). Modeling of hybrid stepper motor finalized to the optimization of the holding torque. Proceedings - 2021 IEEE Workshop on Electrical Machines Design, Control and Diagnosis, WEMDCD 2021, 107-112. https://doi.org/10.1109/WEMDCD51469.2021.9425647

[19] Bjekić, M.; Milovanović, A. \& Koprivica, B. (2015). Prediction of pull-in and pull-out torque characteristics of the permanent magnet step motor, Revue Roumaine des Sciences Techniques Serie Electrotechnique et Energetique, Vol. 60, No. 1, pp. 29-38.

[20] https://marlinfw.org/, (2021), Home | Marlin Firmware (2021)., Accessed on: 2021-04-19

[21] Scheffel, R. M.; Fröhlich, A. A. \& Silvestri, M. (2021). Automated fault detection for additive manufacturing using vibration sensors. International Journal of Computer Integrated Manufacturing, Vol. 34, No. 5, pp. 500-514. https://doi.org/10.1080/0951192X.2021.1901316

[22] https://octoprint.org/, (2021), OctoPrint.org, Accessed on: 2021-04-19

[23] Cekic, A.; Muhamedagic, K.; Begic-Hajdarevic, D. \& Djuzo, N. (2020). Effect of Process Parameters on Dimensional Accuracy and Tensile Strength of FDM Printed Parts, Proceedings of the 31st DAAAM International Symposium, pp.0066-0071, B. Katalinic (Ed.), Published by DAAAM International, ISBN 978-3-902734-29-7, ISSN 1726-9679, Vienna, Austria DOI: 10.2507/31st.daaam.proceedings.009

[24] Madlener, K.; Frey, B. \& Ciezki, H. K. (2009). Generalized reynolds number for non-newtonian fluids, Progress in Propulsion Physics, 1, pp. 237-250. doi: 10.1051/EUCASS/200901237.

[25] Fang, Q. \& Hanna, M. A. (1999). Rheological properties of amorphous and semicrystalline polylactic acid polymers, Industrial Crops and Products, Vol. 10, No. 1, pp. 47-53. doi: 10.1016/S0926-6690(99)00009-6. 